\title{
RÉNYI-WEHRL ENTROPIES AS MEASURES OF LOCALIZATION IN PHASE SPACE
}

\author{
SVEN GNUTZMANN AND KAROL ŻYCZKOWSKI
}

\begin{abstract}
We generalize the concept of the Wehrl entropy of quantum states which gives a basis-independent measure of their localization in phase space. We discuss the minimal values and the typical values of these Rényi-Wehrl entropies for pure states for spin systems. According to Lieb's conjecture the minimal values are provided by the spin coherent states. Though Lieb's conjecture remains unproven, we give new proofs of partial results that may be generalized for other systems. We also investigate random pure states and calculate the mean Rényi-Wehrl entropies averaged over the natural measure in the space of pure quantum states.
\end{abstract}

\section{INTRODUCTION}

Analysis of localization properties of eigenstates of a (chaotic) quantum mechanical system has lead to a lot of insights on the behavior of classically chaotic as well as disordered quantum systems. The localization properties are usually characterized by their eigenvector distribution [1, 2, 3, 4, the entropic localization length [5] or the (inverse) participation ratio [6]. All these quantities, however, are based on the expansion of an eigenstate in some given orthonormal eigenbasis, which mostly can be chosen arbitrarily. If one chooses (with some bad will) the eigenbasis of the system, all these quantities carry no information whatsoever.

Less known but very useful are measures of localization that do not depend on some arbitrary choice. These can be defined using generalized coherent states [7], which provide a connection of the quantum dynamics to the classical phase space. They have been defined canonically for several examples of classical phase spaces using the algebraic construction of Perelomov and Gilmore [7, 8] and provide a representation of a quantum mechanical state by a positive normalized and bounded function on phase space - the so called Husimi function [9]. Let us mention in passing that the Husimi function was successfully applied to study dynamical properties of quantized chaotic systems 10, 11].

The Wehrl entropy has been defined as measure of localization of the Husimi function 12, 13. This has the advantage of being independent of the choice of some orthonormal basis [14]. Probably even more important is the fact that localization properties of quantum mechanical states are measured in the corresponding classical phase space and can be connected to the classical dynamics [15, 16, 17]. Generalized measures of localization are the moments of the Husimi function or Rényi-Wehrl entropies. The second moment has recently ben proposed as a definition of complexity of quantum states [18]

The most localized quantum mechanical states are those which minimize the Wehrl entropy. For Glauber coherent states of a harmonic oscillator [19, 20, 21 Wehrl conjectured and Lieb proved that the state minimizing the entropy is again 
a coherent state 22]. Lieb also conjectured that a similar statement should be true for the angular momentum (spin) coherent states [22]. This conjecture remains unproven so far. There are two ways to generalize the conjectures of Wehrl and Lieb. The first is to generalize it to all systems which allow for Gilmore-Perelomov coherent states. Secondly one may generalize the measure of localization itself (generalized Rényi-Wehrl entropies). In this work we discuss the latter generalization and show that the accordingly generalized Lieb-Wehrl conjecture for the coherent states can be proved for some of the generalized entropies.

This paper is organized as follows. After a very brief introduction to Perelomov coherent states we define in section 2 the Rényi-Wehrl entropies and present the generalized Lieb-Wehrl conjectures. In section 3 we compute the generalized Wehrl entropy for the $S U(2)$ coherent states and prove that they correspond to local minima of these entropies. Moreover, we define the entropy reducing maps and using this notion we provide a new proof of the generalized Lieb conjecture for the Rényi-Wehrl entropies of an integral order. The action of rotations on the Bargmann function, used in the proof, is described in Appendix A. In section 4 we compute the mean Rényi-Wehrl entropy obtained by averaging over the natural measure on the space of pure states. The paper is concluded in section 5 .

\section{Generalized RÉNYi-Wehrl Entropies}

2.1. Gilmore-Perelomov coherent states. The construction of Gilmore-Perelomov coherent states has been described in 18, 7. Spin coherent states, related to the rotation group $S U(2)$, have been introduced in 23, 24]. Coherent states for other compact groups $(S U(3)$ and $S U(n))$ have been recently discussed [25, 26, 27]. Here we only give some of the general properties. In section 3.1 we provide a more detailed account on spin coherent states.

Let $\mathcal{M}$ be the classical phase space of some physical system and $V$ the Hilbert space of the quantized system. The coherent states of such a system form a set of normalized states $\{|\gamma\rangle\}_{\gamma \in \mathcal{M}} \subset V$ with the following properties:

\section{CS.I}

There is a one-to-one mapping $\mathcal{M} \longrightarrow V: \gamma \mapsto|\gamma\rangle$, such that a unique vector in Hilbert space corresponds to each point in the classical phase space.

\section{CS.II}

For some set $\left\{O_{i}\right\}$ of observables the expectation values $\left\langle\gamma\left|O_{i}\right| \gamma\right\rangle$ match the values of the corresponding classical observable at the point $\gamma$ in phase space. This condition can only be fulfilled if the observables $O_{i}$ build a small set of well chosen operators. This set should be large enough to determine uniquely the point $\gamma$ in phase space from the expectation values.

\section{CS.III}

The coherent states minimize the Heisenberg uncertainty among all normalized states. By this property coherent states are in a sense the most classical quantum states.

\section{CS.IV}

There exist a resolution of unity in coherent states, i.e. the set of coherent states is (over)complete.

In the first three conditions the coherent states are defined as a subset of Hilbert space such that this subset is equivalent to the classical phase space in a physically (and mathematically) reasonable way. The condition CS.IV needs additional 
explanation. Being a continuum of normalized states the coherent states cannot form an orthogonal basis - in general the scalar product of two different coherent states does not vanish. Still they satisfy a completeness relation in the form of a resolution of unity

$$
\mathbb{I}=c \int_{\mathcal{M}} d \mu(\gamma)|\gamma\rangle\langle\gamma|
$$

where $\mathbb{I}$ is the identity operator, $d \mu(\gamma)$ denotes a volume element in the phase space (canonically defined by its symplectic structure) and $c$ stands for a normalization constant. This looks much like the completeness relation for an orthonormal basis, in which the sum has been replaced by an integral over the phase space. For this reason the set of coherent states is often said to be overcomplete.

For a large class of classical phase spaces the set of coherent states can be constructed following the group theoretic approach of Perelomov and Gilmore [7, 87. Its main ingredient is a Lie group $G$ and an irreducible representation of that Lie group in some Hilbert space $V$. This construction is known so far for certain classes of Lie groups, including the physically most relevant groups [7]. The coherent states are obtained by action of the Lie group $G$ on an initial state $|0\rangle$ chosen according to condition CS.III,

$$
|\gamma\rangle=g(\gamma)|0\rangle
$$

where $g(\gamma) \in G$. It can be shown that the classical phase space is equivalent to $G / H$, where $H$ is the subgroup of $G$ that leaves $|0\rangle$ invariant up to some phase, $\left(h|0\rangle=e^{i \phi}|0\rangle\right.$ for all $\left.h \in H\right)$. The relevant set of observables (condition CS.II) is given by the generators of the group. In the case of a spin the Lie group is $G=S U(2)$, whose generators satisfy the commutation relations of the components of an angular momentum operator.

One straightforward way to describe quantum mechanics in phase space is to use the overlap of a state with a coherent state, $\psi(\gamma)=\langle\gamma \mid \psi\rangle$. The square of the absolute value of this function is called the Husimi function [9] (or $Q$-function)].

$$
\mathcal{H}_{|\psi\rangle}(\gamma)=|\langle\gamma \mid \psi\rangle|^{2} \text {. }
$$

One can easily show that the Husimi function is non-negative, bounded?

$$
0 \leq \mathcal{H}_{|\psi\rangle}(\gamma) \leq 1
$$

and normalized

$$
c \int_{\mathcal{M}} d \mu(\gamma) \mathcal{H}_{|\psi\rangle}(\gamma)=\langle\psi \mid \psi\rangle=1
$$

One is tempted to think of the Husimi function as a probability density on the phase space. However the rules for calculating expectation values of some observable using the Husimi function are non-classical. Still the label quasiprobability has been introduced in this context and in a properly defined classical limit $c \mathcal{H}_{|\psi\rangle}$ becomes a usual classical probability distribution.

Due to the overcompleteness of the coherent states one can actually show that the Husimi function determines the state $|\psi\rangle$ up to some phase. This contrasts the properties of the expansion of a state in an orthonormal basis, in which the phase

\footnotetext{
1 In the mathematical literature the Husimi function is also called covariant or Wick symbol.

${ }^{2}$ Actually there are many ways to define functions on phase space that represent a quantum state completely. The Husimi function is the only one which is non-negative and bounded.
} 
information is lost by squaring the expansion coefficients. This is actually the reason, why expansions in coherent states are much more powerful than expansions in some orthonormal basis for defining useful measures of localization.

In the following we will restrict ourselves to pure states. Generalizations to mixed states are straightforward. For the statistical operator $\rho=\sum p_{i}\left|\psi_{i}\right\rangle\left\langle\psi_{i}\right|$ the Husimi function is $\mathcal{H}_{\rho}(\gamma)=\langle\gamma|\rho| \gamma\rangle$. Again it is possible to reconstruct the statistical operator $\rho$ from its Husimi function $\mathcal{H}_{\rho}(\gamma)$.

2.2. Definition of the generalized Rényi-Wehrl entropies. Wehrl has defined the phase space entropy of a quantum state $|\psi\rangle$ as the continuous (Boltzmann) entropy of its Husimi function 12,

$$
S_{|\psi\rangle}=-c \int_{\mathcal{M}} d \mu(\gamma) \mathcal{H}_{|\psi\rangle} \log \mathcal{H}_{|\psi\rangle} .
$$

The generalized Wehrl entropies carry the Rényi index $q>0$ and are defined as

$$
S_{|\psi\rangle}^{(q)}=\frac{1}{1-q} \log W_{|\psi\rangle}^{(q)},
$$

with the 'moment' functions

$$
W_{|\psi\rangle}^{(q)}=c \int_{\mathcal{M}} d \mu(\gamma)\left(\mathcal{H}_{|\psi\rangle}\right)^{q} .
$$

In the limit $q \rightarrow 1$ one gets back the original Wehrl entropy,

$$
\lim _{q \rightarrow 1} S_{|\psi\rangle}^{(q)}=S_{|\psi\rangle}
$$

so for consistency the Wehrl entropy $S_{|\psi\rangle}$ will be denoted by $S_{|\psi\rangle}^{(1)}$.

The Rényi-Wehrl entropy is a non-increasing function of the parameter $q$

$$
q_{2}>q_{1} \quad \Longrightarrow \quad S_{|\psi\rangle}^{\left(q_{2}\right)} \leq S_{|\psi\rangle}^{\left(q_{1}\right)} \text {. }
$$

For compact phase spaces one can put $\int_{\mathcal{M}} d \mu(\gamma)=1$. Then $c=N$ is the finite dimension of Hilbert space. The function

$$
\mathcal{J}_{|\psi\rangle}(\gamma)=1-\mathcal{H}_{|\psi\rangle}(\gamma)
$$

is also bounded $0 \leq \mathcal{J}_{|\psi\rangle}(\gamma) \leq 1$ and normalizable. It thus makes sense to define the following dual Wehrl entropy

$$
Z_{|\psi\rangle}=-\frac{N}{N-1} \int_{\mathcal{M}} d \mu(\gamma) \mathcal{J}_{|\psi\rangle} \log \mathcal{J}_{|\psi\rangle}
$$

and its Rényi-Wehrl generalization

$$
Z_{|\psi\rangle}^{(q)}=\frac{1}{1-q} \log Y_{|\psi\rangle}^{(q)}
$$

with the dual moments

$$
Y_{|\psi\rangle}^{(q)}=\frac{N}{N-1} \int_{\mathcal{M}} d \mu(\gamma)\left(\mathcal{J}_{|\psi\rangle}\right)^{q}
$$

Making use of the monotonicity of the logarithm and the definition of the entropies it is easy to show that for any two pure states $\left|\psi_{1}\right\rangle$ and $\left|\psi_{2}\right\rangle$ and any $q>1$ one has

$$
W_{\left|\psi_{1}\right\rangle}^{(q)}>W_{\left|\psi_{2}\right\rangle}^{(q)}\left(Y_{\left|\psi_{1}\right\rangle}^{(q)}>Y_{\left|\psi_{2}\right\rangle}^{(q)}\right) \quad \Leftrightarrow \quad S_{\left|\psi_{1}\right\rangle}^{(q)}<S_{\left|\psi_{2}\right\rangle}^{(q)} \quad\left(Z_{\left|\psi_{1}\right\rangle}^{(q)}<Z_{\left|\psi_{2}\right\rangle}^{(q)}\right)
$$

while for $q<1$ one has $W_{\left|\psi_{1}\right\rangle}^{(q)}>W_{\left|\psi_{2}\right\rangle}^{(q)} \Leftrightarrow S_{\left|\psi_{1}\right\rangle}^{(q)}>S_{\left|\psi_{2}\right\rangle}^{(q)}$. 
All the entropies $S^{(q)}$ and $Z^{(q)}$ provide measures of localization of a quantum state in phase space. Since the participation ratio is a very useful entropy-like characteristic of uniformity of a probability distribution, we also define its analogue in phase space: the Wehrl participation ratio,

$$
R_{|\psi\rangle}=\frac{1}{W_{|\psi\rangle}^{(2)}}=\exp S_{|\psi\rangle}^{(2)}
$$

and its dual

$$
T_{|\psi\rangle}=\frac{1}{Y_{|\psi\rangle}^{(2)}}=\exp Z_{|\psi\rangle}^{(2)}
$$

All these quantities are invariant under the action of the Lie group $G$, e.g.

$$
S_{|\psi\rangle}^{(q)}=S_{g|\psi\rangle}^{(q)}
$$

where $g \in G$. This ensures that all these entropies do depend only on the shape of the Husimi function and not on the position of a point in phase space, in vicinity of which they are localized.

2.3. The generalized Lieb-Wehrl conjecture. For compact phase space the Rényi-Wehrl entropies are bounded $S_{|\psi\rangle}^{(q)} \leq \log N$ (with the dimension of Hilbert space $N$ ). This upper bound corresponds to a mixed state with statistical operator $\rho=\frac{1}{N} \mathbb{I}$. The maximal value $S_{\max }^{(q)}$ for the Rényi-Wehrl entropies among pure states is strictly smaller. However, neither the exact value $S_{\max }^{(q)}$ nor the states corresponding to it are known in general.

The Rényi-Wehrl entropies are positive, $S_{|\psi\rangle}^{(q)}>0$. The minimal value $S_{\text {min }}^{(q)}$ can be expected for coherent states. For $S_{|\psi\rangle}$ of a harmonic oscillator this was conjectured by Wehrl [12] and proved by Lieb [22]. Lieb extended the conjecture to spin $(S U(2))$ coherent states, which remains unproven up till now. It is tempting to expect that the following generalization is true.

Conjecture 1 (Lieb-Wehrl, generalized). The minimal value $S_{\mathrm{min}}^{(q)}$ of the RényiWehrl entropies for $q>0$ are obtained for coherent states

$$
S_{\mathrm{min}}^{(q)}=S_{\mathrm{coh}}^{(q)}
$$

For compact phase spaces the same is true for the dual Rényi-Wehrl entropies $Z_{\min }^{(q)}=Z_{\mathrm{coh}}^{(q)}$.

\section{RÉNYI-WEHRL ENTROPIES FOR SPIN COHERENT STATES}

3.1. Phase space quantum mechanics of a spin and $\mathbf{S U}(2)$ coherent states. As it is well known, an angular momentum operator may be described by three operators $J_{z}, J_{+}=J_{x}+i J_{y}, J_{-}=J_{x}-i J_{y}$, fulfilling the commutation relations $\left[J_{+}, J_{-}\right]=2 J_{z}$ and $\left[J_{z}, J_{ \pm}\right]= \pm J_{ \pm}$. They are the generators of the Lie group $S U(2)$. The Hilbert space $V_{j}$ has dimension $N=2 j+1$, where $j$ is the spin quantum number defined by $J^{2}=j(j+1) \mathbb{I}$. An orthonormal basis of $V_{j}$ is given by the eigenstates of the $z$-th component of the spin, $J_{z}|m\rangle=m|m\rangle$ with $m=-j,-j+1, \ldots, j-1, j$. The classical phase space for a spin is the Bloch sphere $S^{2}$. For convenience we 
choose stereographic coordinates on the sphere. A point on the sphere is then given by one complex number $\gamma \in \mathbb{C}$ and has polar coordinates given by

$$
\cos \theta=\frac{1-|\gamma|^{2}}{1+|\gamma|^{2}} \quad \text { and } \quad e^{i \phi}=\frac{\bar{\gamma}}{|\gamma|} .
$$

The 'north-pole' has the coordinate $\gamma=0$ and the 'south-pole' can only be reached in the limit $|\gamma| \rightarrow \infty$.

The state $|j\rangle$ is localized at the north-pole of the sphere and can be shown to minimize an uncertainty relation [28]. Coherent states $|\gamma\rangle$ are obtained by rotating the state $|j\rangle$ by some rotation matrix $\mathcal{R}(\gamma) \in S U(2)$. Equivalently one may define first a set of non-normalized coherent states by

$$
\mid \gamma)=e^{\gamma J_{-}}|j\rangle
$$

Their normalized counterparts are, (note the difference in brackets used)

$$
\left.|\gamma\rangle=\frac{1}{\sqrt{\mathcal{K}(\gamma)}} \mid \gamma\right) \quad \text { with } \quad \mathcal{K}(\gamma)=(\gamma \mid \gamma)=\left(1+|\gamma|^{2}\right)^{2 j}
$$

Obviously the state $|j\rangle$ is a coherent state with $\gamma=0$.

The resolution of unity is given by

$$
\mathbb{I}=(2 j+1) \int_{S^{2}} d \mu(\gamma)|\gamma\rangle\left\langle\gamma\left|=(2 j+1) \int_{S^{2}} d \mu(\gamma) \frac{1}{\mathcal{K}(\gamma)}\right| \gamma\right)(\gamma \mid
$$

with the rotation-invariant area element (Haar measure)

$$
d \mu(\gamma)=\frac{1}{\pi\left(1+|\gamma|^{2}\right)^{2}} d \operatorname{Re}(\gamma) d \operatorname{Im}(\gamma)
$$

on the Bloch sphere.

Any state $|\psi\rangle$ is uniquely determined by its overlap with coherent states. Thus, one may define the so called Bargmann functions on the sphere

$$
\gamma \mapsto \psi(\gamma)=\langle\psi| \gamma)=\left\langle\psi\left|e^{\gamma J_{-}}\right| j\right\rangle .
$$

As $J_{-}^{k}|j\rangle=0$ for $k>2 j$ the Bargmann function is a finite polynomial in $\gamma$

$$
\psi(\gamma)=\sum_{k=0}^{2 j} f_{k} \gamma^{k}
$$

with some complex coefficients $f_{k}$. The scalar product of two states can be written in terms of their Bargmann functions as

$$
\left\langle\psi_{1} \mid \psi_{2}\right\rangle=(2 j+1) \int d \mu(\gamma) \mathcal{K}^{-1}(\gamma) \psi_{1}(\gamma) \overline{\psi_{2}(\gamma)} .
$$

The normalization of a state then leads to the condition $\sum_{k=0}^{2 j}\left(\begin{array}{c}2 j \\ k\end{array}\right)^{-1}\left|f_{k}\right|^{2}=1$. As the monomials $\gamma^{k}(k=0,1,2, \ldots)$ build an orthogonal (but not normal) basis one also has $\left|f_{k}\right|^{2} \leq\left(\begin{array}{c}2 j \\ k\end{array}\right)$. If the state is a normalized coherent state, $|\psi\rangle=$ $\left.\mathcal{K}\left(\gamma^{\prime}\right)^{-1 / 2} \mid \gamma^{\prime}\right)$, the corresponding Bargmann function reads

$$
\left.\psi(\gamma)=\left\langle\gamma^{\prime}\right| \gamma\right)=\mathcal{K}\left(\gamma^{\prime}\right)^{-1 / 2}\left(\gamma^{\prime} \mid \gamma\right)=\frac{\left(1+\overline{\gamma^{\prime}} \gamma\right)^{2 j}}{\left(1+\left|\gamma^{\prime}\right|^{2}\right)^{j}}
$$

The limit $\left|\gamma^{\prime}\right| \rightarrow \infty$ corresponds to the Bargmann function for a coherent state on the south pole. While $\left(\gamma^{\prime} \mid\right.$ does not exist in this limit, its Bargmann function is well behaved and gives $\mathcal{K}\left(\gamma^{\prime}\right)^{-1 / 2}\left(\gamma^{\prime} \mid \gamma\right) \longrightarrow \gamma^{2 j} e^{-4 i j \alpha}$, where the phase $\alpha$ is defined 
by $\gamma^{\prime}=\left|\gamma^{\prime}\right| e^{i \alpha}$. The Bargmann function for a coherent state on the 'north pole' is simply constant, $\psi(\gamma)=\left.\mathcal{K}\left(\gamma^{\prime}\right)^{-1 / 2}\left(\gamma^{\prime} \mid \gamma\right)\right|_{\gamma^{\prime}=0}=1$.

Every state $|\psi\rangle$ is defined up to some phase factor by the $2 j$ zeroes $\gamma_{k}^{(0)}$ of its Bargmann function by the stellar representation 29, 30, 31]

$$
\psi(\gamma)=\mathcal{N}^{-1} \prod_{k=1}^{2 j-m}\left(\gamma-\gamma_{k}^{(0)}\right)
$$

where $\mathcal{N}$ is some normalization constant and $m$ is the number of zeroes at the south pole..$^{3}$ A coherent state is characterized by one $2 j$-fold degenerate zero of the Bargmann function.

The Husimi function of a pure state is closely related with its Bargmann function,

$$
\mathcal{H}_{\psi}(\gamma)=\frac{|\psi(\gamma)|^{2}}{\mathcal{K}(\gamma)}
$$

Just as the Bargmann function the Husimi function of a pure state is completely determined by its $2 j$ zeroes. Therefore, the zeroes of the Husimi and the Bargamann functions coincide for any pure state.

From Schrödinger's equation $i \hbar \frac{d}{d t}|\psi(t)\rangle=H|\psi(t)\rangle$ with the hermitian Hamiltonian $H$ one gets an evolution equation for the Bargmann function, $\psi(\gamma ; t)=\psi(t)(\gamma)$,

$$
i \hbar \frac{d}{d t} \overline{\psi(\gamma ; t)}=(2 j+1) \int d \mu\left(\gamma^{\prime}\right) \mathcal{K}\left(\gamma^{\prime}\right)^{-1} H\left(\bar{\gamma}, \gamma^{\prime}\right) \overline{\psi\left(\gamma^{\prime} ; t\right)}
$$

with

$$
H\left(\bar{\gamma}, \gamma^{\prime}\right)=\left(\gamma|H| \gamma^{\prime}\right)=\sum_{k, k^{\prime}=0}^{2 j} H_{k k^{\prime}} \bar{\gamma}^{k} \gamma^{k^{\prime}} .
$$

The hermiticity of $H$ is reflected in $\overline{H\left(\bar{\gamma}, \gamma^{\prime}\right)}=H\left(\bar{\gamma}^{\prime}, \gamma\right)$ and $H_{k k^{\prime}}=\overline{H_{k^{\prime} k}}$. For the time dependence of the Husimi function this gives

$$
i \hbar \frac{d}{d t} \mathcal{H}_{|\psi\rangle}(\gamma ; t)=\int d \mu\left(\gamma^{\prime}\right) \frac{2 j+1}{\mathcal{K}(\gamma) \mathcal{K}\left(\gamma^{\prime}\right)}\left(\psi(\gamma) H\left(\bar{\gamma}, \gamma^{\prime}\right) \overline{\psi\left(\gamma^{\prime}\right)}-\psi\left(\gamma^{\prime}\right) H\left(\bar{\gamma}^{\prime}, \gamma\right) \overline{\psi(\gamma)}\right) .
$$

The integral equations (31) and (33) can in general be transformed into equivalent differential equations. For our purposes the integral form is better suited.

3.2. Rényi-Wehrl entropies for spins. It is usually quite difficult to give a closed expression for the integrals $W_{|\psi\rangle}^{(q)}$ and $Y_{|\psi\rangle}^{(q)}$ for a general state $|\psi\rangle$. For the Wehrl entropy $S_{|\psi\rangle}$ the stellar representation helps and an explicit expression was given by Lee [32, 33].

For a coherent state the integrals are straightforward (using $\mathcal{H}=\mathcal{K}^{-1}$ for the coherent state on the north pole $\gamma=0$ ) and all measures of localization can be

\footnotetext{
${ }^{3}$ The limit when one of the $\gamma_{k}^{(0)}$ approaches infinity is well defined and reduces the rank of the polynomial.
} 
expressed analytically

$$
\begin{aligned}
W_{\mathrm{coh}}^{(q)} & =\frac{2 j+1}{2 q j+1}, & Y_{\mathrm{coh}}^{(q)} & =\frac{\Gamma(q+1) \Gamma\left(\frac{1}{2 j}+2\right)}{\Gamma\left(q+1+\frac{1}{2 j}\right)}, \\
S_{\mathrm{coh}} & =\frac{2 j}{2 j+1}, & Z_{\mathrm{coh}} & =\Psi\left(\frac{4 j+1}{2 j}\right)+\gamma-1, \\
R_{\mathrm{coh}} & =\frac{4 j+1}{2 j+1}, & T_{\mathrm{coh}} & =\frac{4 j+1}{4 j} .
\end{aligned}
$$

Here $\Psi(x)$ denotes the Digamma function and $\gamma=0.5772 \ldots$ is the Euler constant. For the eigenstates $|m\rangle(m=-j, \ldots, j)$ of $J_{z}$ one obtains

$$
\begin{aligned}
W_{|m\rangle}^{(q)}= & \frac{2 j+1}{2 q j+1}\left(\begin{array}{c}
2 j \\
j-m
\end{array}\right)^{q}\left(\begin{array}{c}
2 q j \\
q(j-m)
\end{array}\right)^{-1}, \\
S_{|m\rangle}= & \frac{2 j}{2 j+1}-\log \left(\begin{array}{c}
2 j \\
j-m
\end{array}\right)+2 j \Psi(2 j+1)+ \\
& -(j+m) \Psi(j+m+1)-(j-m) \Psi(j-m+1), \\
R_{|m\rangle}= & \frac{4 j+1}{2 j+1}\left(\begin{array}{c}
4 j \\
2(j-m)
\end{array}\right)\left(\begin{array}{c}
2 j \\
j-m
\end{array}\right)^{-2} .
\end{aligned}
$$

For $m=-j$ or $m=j$ this reproduces the results for coherent states. For $m=0$ the state is localized on the equator of the Bloch sphere. For large $N=2 j+1$ the Wehrl entropy of this state increases like $S_{|m=0\rangle} \sim \frac{1}{2} \log N+$ const. As it was shown in 17] this behaviour is typical for the eigenstates of an integrable map on the sphere. However, for a typical (random) state the Wehrl entropy grows as $S_{|\psi\rangle} \sim$ $\log N$-const, with a positive constant. Such behaviour is found for the eigenstates of quantized chaotic maps like the periodically kicked top. Asymptotically, both results differ by a factor of two. This reflects the fact that the eigenstates of chaotic systems are delocalized over entire sphere, while the eigenstates of integrable systems typically cover a band along a (possibly deformed) circle on the sphere.

For integer values of the Rényi parameter, $q=2,3, \ldots(q=1$ is trivial and gives the norm), it is possible to give an explicit result for the moments

$$
W_{|\psi\rangle}^{(q)}=\sum_{m=0}^{2 q j} \frac{2 j+1}{2 q j+1}\left(\begin{array}{c}
2 q j \\
m
\end{array}\right)^{-1}\left|\sum_{i_{1}, i_{2}, \ldots, i_{q}} \prod_{k=1}^{q} f_{i_{k}}\right|^{2},
$$

where the coefficients $f_{k}$ are given by (26) and the inner sum goes from 0 to $2 j$ for each $i_{k}$ with the restriction $\sum_{k=1}^{q} i_{k}=m$.

3.3. Time dependence of the Rényi-Wehrl entropies. If a time-dependent state $|\psi(t)\rangle=e^{-i \frac{H t}{\hbar}}|\psi(0)\rangle$ obeys the Schrödinger's equation, the Rényi-Wehrl entropies $S_{|\psi(t)\rangle}^{(q)}$ will generally vary in time. However, if the Hamiltonian is of the form $H=a J_{z}+b J_{+}+\bar{b} J_{-}(a \in \mathbb{R}, c \in \mathbb{C})$, the evolution of a state corresponds to a rotation $e^{-i \frac{H t}{\hbar}}=\mathcal{R} \in S U(2)$, the coherent states remain coherent and all RényiWehrl entropies are constant in time. In general $S_{|\psi(t)\rangle}^{(q)}$ is quasiperiodic. The rate 
RÉNYI-WEHRL ENTROPIES AS MEASURES OF LOCALIZATION IN PHASE SPACE 9

of change of the entropies and moments is given by

$$
\begin{aligned}
\frac{d}{d t} S_{|\psi(t)\rangle} & =-(2 j+1) \int d \mu(\gamma) \log \mathcal{H}_{|\psi\rangle}(\gamma ; t)\left[\frac{d}{d t} \mathcal{H}_{|\psi\rangle}(\gamma ; t)\right], \\
\frac{d}{d t} S_{|\psi(t)\rangle}^{(q)} & =\frac{1}{(1-q) W_{|\psi(t)\rangle}^{(q)}}\left[\frac{d}{d t} W_{|\psi(t)\rangle}^{(q)}\right], \\
\frac{d}{d t} W_{|\psi(t)\rangle}^{(q)} & =q(2 j+1) \int d \mu(\gamma)\left(\mathcal{H}_{|\psi\rangle}(\gamma ; t)\right)^{q-1}\left[\frac{d}{d t} \mathcal{H}_{|\psi\rangle}(\gamma ; t)\right], \\
\frac{d}{d t} Z_{|\psi(t)\rangle} & =\frac{2 j+1}{2 j} \int d \mu(\gamma) \log \left(1-\mathcal{H}_{|\psi\rangle}(\gamma ; t)\right)\left[\frac{d}{d t} \mathcal{H}_{|\psi\rangle}(\gamma ; t)\right], \\
\frac{d}{d t} Z_{|\psi(t)\rangle}^{(q)} & =\frac{1}{(1-q) Y_{|\psi(t)\rangle}^{(q)}}\left[\frac{d}{d t} Y_{|\psi(t)\rangle}^{(q)}\right], \\
\frac{d}{d t} Y_{|\psi(t)\rangle}^{(q)} & =-q \frac{2 j+1}{2 j} \int d \mu(\gamma)\left(1-\mathcal{H}_{|\psi\rangle}(\gamma ; t)\right)^{q-1}\left[\frac{d}{d t} \mathcal{H}_{|\psi\rangle}(\gamma ; t)\right] .
\end{aligned}
$$

Pure states, for which these time derivatives vanish for every Hamiltonian $H$ will be called extremal. It will be shown below that the coherent states belong to this class. For an extremal state it is also interesting to calculate the second derivatives which we only need for the moment functions

$$
\begin{aligned}
\frac{d^{2}}{d t^{2}} W_{|\psi(t)\rangle}^{(q)}= & q(q-1)(2 j+1) \int d \mu(\gamma)\left(\mathcal{H}_{|\psi\rangle}(\gamma ; t)\right)^{q-2}\left[\frac{d}{d t} \mathcal{H}_{|\psi\rangle}(\gamma ; t)\right]^{2} \\
& +q(2 j+1) \int d \mu(\gamma)\left(\mathcal{H}_{|\psi\rangle}(\gamma ; t)\right)^{q-1}\left[\frac{d^{2}}{d t^{2}} \mathcal{H}_{|\psi\rangle}(\gamma ; t)\right] \\
\frac{d^{2}}{d t^{2}} Y_{|\psi(t)\rangle}^{(q)}= & q(q-1) \frac{2 j+1}{2 j} \int d \mu(\gamma)\left(1-\mathcal{H}_{|\psi\rangle}(\gamma ; t)\right)^{q-2}\left[\frac{d}{d t} \mathcal{H}_{|\psi\rangle}(\gamma ; t)\right]^{2} \\
& -q(2 j+1) \int d \mu(\gamma)\left(1-\mathcal{H}_{|\psi\rangle}(\gamma ; t)\right)^{q-1}\left[\frac{d^{2}}{d t^{2}} \mathcal{H}_{|\psi\rangle}(\gamma ; t)\right]
\end{aligned}
$$

3.4. The generalized Lieb-Wehrl conjecture for spins. The generalized LiebWehrl conjecture stated in section 2.3 is not easy to prove even in the simplest case of the $S U(2)$ coherent states and the Bloch sphere. The original conjecture of Lieb concerning the Wehrl entropy $S_{|\psi\rangle}$ (i.e. the case $q=1$ ) has been proved only for small spin quantum numbers $j=1$ and $j=3 / 2$ [33, 34 $]^{\prime}$. It is however possible to show that all states sufficiently near to a coherent state have larger Rényi-Wehrl entropies.

Theorem 1. The coherent states minimize the entropies $S^{(q)}$ and $Z^{(q)}$ locally for any $q>0$. The moments $W^{(q)}$ and $Y^{(q)}$ are locally maximized if $q>1$ and locally minimized if $0<q<1$.

Proof. In the Hilbert space $V_{j}$ any two normalized pure states can be transformed into another by a unitary operator $U,\left|\psi_{1}\right\rangle=U\left|\psi_{2}\right\rangle$. Also, for any unitary operator on $V_{j}$ there is a hermitian operator $H$ such that $U=e^{-i H}$. In this proof we think of $H$ as the Hamiltonian of a dynamical quantum system and $|0\rangle$ as the initial condition. Now for any state $|\psi\rangle$ in a neighborhood of the coherent state on the

\footnotetext{
${ }^{4}$ The case $j=1 / 2$ is trivial as every pure state is a coherent state.
} 
north pole, $|0\rangle$, there is a Hamiltonian, such that after some time $t$ the dynamical state of the system is $|\psi\rangle$.

It suffices to prove the statement on the moment functions. Due to invariance under rotation one may choose the coherent state on the north pole $(\gamma=0)$ with the Husimi function $\mathcal{H}=\frac{1}{\left(1+|\gamma|^{2}\right)^{2 j}}$. Choose this state as initial state for Schrödinger's equation with some arbitrary Hamiltonian $H$. Using equations (43) and (46) we conclude that at $t=0$ the first derivative of these moments vanish for every Hamiltonian $H$. In more tedious calculations for the second derivatives at $t=0$ one uses equations (47) and (48). For $q>1(0<q<1)$ the second derivatives of the moments are either negative (positive) or they vanish. The Hamiltonians $H$ which lead to the vanishing second derivative either have the coherent state on the north pole as an eigenstate or are locally equivalent to a rotation. Thus in all cases with vanishing second derivative the state remains within the manifold of coherent states for infinitesimal times.

To complete the proof of the Lieb-Wehrl conjecture for the Bloch sphere one needs to show that $S_{|\psi\rangle}^{(q)}>S_{\text {coh }}^{(q)}$ for any non-coherent state $|\psi\rangle$.

Definition 1. We call a general norm-preserving map $\mathcal{F}: V_{j} \rightarrow V_{j}$ and $\mathcal{F}:|\psi\rangle \mapsto$ $\mathcal{F}(|\psi\rangle)$ with $\mathcal{F}(\alpha|\psi\rangle)=\alpha \mathcal{F}(|\psi\rangle)$ entropy reducing if we have $S_{|\psi\rangle}^{(q)} \geq S_{\mathcal{F}(|\psi\rangle)}^{(q)}$ for every $|\psi\rangle \in V_{j}$.

The map $\mathcal{F}$ need not be one-to-one or linear, so it need not have any physical relevance. However, finding such an entropy reducing map would be a step toward the proof of the Lieb conjecture. Loosely speaking we are looking for a sequence of contracting transformations in the space of all pure states (complex projective space $\left.\mathbb{C} P^{N-1}\right)$, with an attractor at the set of coherent states, $\left(\mathbb{C} P^{1} \approx S^{2} \subset \mathbb{C} P^{N-1}\right)$.

We shall discuss the following candidates for such entropy reducing maps, defined by their action on the Bargmann function

Let $\mathcal{F}_{1}: V_{j} \rightarrow V_{j}$ be the map defined in the stellar representation by

$$
\psi(\gamma)=\mathcal{N}^{-1} \prod_{k}\left(\gamma-\gamma_{k}^{(0)}\right) \mapsto \mathcal{F}_{1} \circ \psi(\gamma)=\mathcal{N}^{\prime-1} \prod_{k}\left(\gamma-\left|\gamma_{k}^{(0)}\right|\right) .
$$

The geometrical meaning of this map is to rotate all zeroes independently around the $\mathrm{z}$-Axis until they meet the Greenwich meridian defined by $\phi=0$. Rotating the zeroes independently is in general a non-unitary and a noninvertible map. The above map $\mathcal{F}_{1}$ may be supplemented by another map $\mathcal{F}_{1}^{\prime}$, which moves all zeros located along the meridian, into the direction of a certain point belonging to the meridian, say its center at $\theta=\pi / 2$. Then $\mathcal{F}_{1}^{\prime}=\mathcal{F}_{1} \circ \mathcal{R}_{x}(\pi / 2)$ where $\mathcal{R}_{x}(\pi / 2)$ is a quarter rotation around the $\mathrm{x}$-axis and the composition, $\mathcal{F}_{1}^{\prime} \circ \mathcal{F}_{1}=\mathcal{F}_{1} \circ \mathcal{R}_{x}(\pi / 2) \circ \mathcal{F}_{1}$, transforms all zeros of the Husimi function into one point, or in other words, maps any pure state into a coherent state.

Another map $\mathcal{F}_{2}: V_{j} \rightarrow V_{j}$ can similarly be defined for the coefficients

$$
\psi(\gamma)=\sum_{k=0}^{2 j} f_{k} \gamma^{k} \mapsto \mathcal{F}_{2} \circ \psi(\gamma)=\sum_{k=0}^{2 j}\left|f_{k}\right| \gamma^{k} .
$$

There is no geometrical interpretation like before. However, all zeroes of $\mathcal{F}_{2}(|\psi\rangle)$ come in complex conjugate pairs and negative real part. 
Both $\mathcal{F}_{1}$ and $\mathcal{F}_{2}$ are not invariant under unitary rotions $\mathcal{R} \in S U(2)$ of the states

$$
\mathcal{F}_{i} \circ \mathcal{R} \neq \mathcal{R}_{\mathcal{F}_{i}} .
$$

Also, the map $\mathcal{F}_{i} \circ \mathcal{R}$ is entropy reducing if and only if $\mathcal{F}_{i}$ is. In general, any map $\mathcal{F}$ that increases the density of zeroes of the Bargmann function in a certain region of phase space is a candidate for an entropy reducing map. This follows from the observation that a coherent state has only one $2 j$-fold degenerate zero. As such an alternative one could consider another transformation $\mathcal{F}_{3}$, pushing independently each zero into the direction determined by the barycenter of all zeros, $\vec{r}_{B}:=\sum_{i=1}^{N} \vec{r}_{i}$, where $\vec{r}_{i}$ denotes the unit vector pointing to the zero $\gamma_{i}$. In the generic case the radius of the barycenter is nonzero, and its direction distinguishes a certain point in the sphere. This transformation reduces the simplified Monge distance of any pure state to the set of coherent states, which is defined by the minimal sum of distances (measured as angles on the sphere) of all $N$ zeros to any point on the sphere 35.

Not being able to provide a general proof that the maps $\mathcal{F}_{i}$ are entropy reducing, we can give the following result.

Lemma 1. For $q=2,3, \ldots$ and $\left|\psi^{\prime}\right\rangle=\mathcal{F}_{2}(|\psi\rangle)$ one has $S_{\left|\psi^{\prime}\right\rangle}^{(q)} \leq S_{|\psi\rangle}^{(q)}$ or, equivalently, $W_{\left|\psi^{\prime}\right\rangle}^{(q)} \geq W_{|\psi\rangle}^{(q)}$. Equality holds if and only if the phases of the coefficients obey $f_{k}=e^{i \alpha k}$ for some fixed angle $\alpha$.

Proof. It suffices to prove the statement on the moments $W^{(q)}$. In equation (40) the moments were calculated in the form $W_{|\psi\rangle}^{(q)}=\sum_{m=0}^{2 q j} a_{q j m}\left|\sum_{i} z_{i}\right|^{2}$ with coefficients $a_{q j m}>0$.

Now $W_{\left|\psi^{\prime}\right\rangle}^{(q)} \geq W_{\left|\psi^{\prime}\right\rangle}^{(q)}$ follows immediately from $\left|\sum_{i} z_{i}\right|^{2} \leq\left(\sum_{i}\left|z_{i}\right|\right)^{2}$. The statement on equality follows from the condition $\left|\sum_{i} z_{i}\right|^{2}=\left(\sum_{i}\left|z_{i}\right|\right)^{2}$.

Equipped with this lemma we may now state a proof of the generalized Lieb-Wehrl conjecture for the integer values of the Rényi parameter 5 .

Theorem 2. For $q=2,3, \ldots$ the inequalities $S_{\mathrm{coh}}^{(q)} \leq S_{|\psi\rangle}^{(q)}$ and $W_{\mathrm{coh}}^{(q)} \geq W_{|\psi\rangle}^{(q)}$ hold for every state $|\psi\rangle \in V_{j}$ with strict inequality for non-coherent states.

Similarly $Z_{\text {coh }} \leq Z_{|\psi\rangle}$.

Proof. The statements on $S^{(q)}$ and $W^{(q)}$ are equivalent. The statement on $Z_{|\psi\rangle}$ can be reduced to the statements on $W^{(q)}$ using

$$
Z_{|\psi\rangle}=1-\frac{1}{2 j} \sum_{n=2}^{\infty} \frac{W_{|\psi\rangle}^{(n)}}{n(n-1)} .
$$

For any non-coherent state $|\psi\rangle$ described by its Bargmann function $\psi(\gamma)$ we will construct a state $\psi^{\prime}(\gamma)$ such that $W_{|\psi\rangle}^{(q)}<W_{\left|\psi^{\prime}\right\rangle}^{(q)}$, using the entropy reducing map $\mathcal{F}_{2}$ defined in $(50)$ and rotations $\mathcal{R} \in S U(2)$. As $W_{|\psi\rangle}^{(q)}$ is a bounded function on a compact manifold (the projective space $\mathbb{P} V_{j}$ ) and the coherent states provide a local maximum, finding such a state is equivalent to showing that the coherent states are a global maximum and that every global maximum is at a coherent state.

\footnotetext{
${ }^{5}$ An alternative proof of this fact has been already provided by Schupp [33].
} 
In general we have $W_{|\psi\rangle}^{(q)} \leq W_{\mathcal{F}_{2}(|\psi\rangle)}^{(q)}$ with strict inequality if $\mathcal{F}_{2}(|\psi\rangle)$ is a coherent state while $|\psi\rangle$ is not. So there are no noncoherent states that are mapped to a coherent state by $\mathcal{F}_{2}$ without increasing $W^{(q)}$. It follows that we may assume in the following that all coefficients $f_{k}$ of $\psi(\gamma)=\sum_{k=0}^{2 j} f_{k} \gamma^{k}$ are real and nonnegative, $f_{k} \geq 0$ (and we still assume that $\psi(\gamma)$ is not a coherent state). Since $\psi(\gamma)$ does not describe a coherent state, there is no $z \in \mathbb{R}$ such that $f_{k}=\left(\begin{array}{c}2 j \\ k\end{array}\right) \frac{z^{k}}{\left(1+|z|^{2}\right)^{2 j}}$. Further action of $\mathcal{F}_{2}$ cannot increase the value of $W^{(q)}$.

We now show that the combined action of a suitable rotation $\mathcal{R}(\alpha, \phi) \in S U(2)$ as given by $\left(\sqrt[66]{)}\right.$ and $(68)$ followed by $\mathcal{F}_{2}$ gives the desired result. The action of rotations on Bargmann functions is described in the appendix A. It suffices to consider the transformation $\mathcal{R}_{x}:=\mathcal{R}(\alpha=x, \phi=0)$ with real $x$, which represents the rotation around the y-axis. The rotated Bargmann function for an arbitrary $x$ is then given by

$$
\begin{aligned}
\mathcal{R}_{x} \circ \psi(\gamma) & =\frac{(1+x \gamma)^{2 j}}{\left(1+x^{2}\right)^{j}} \psi\left(\frac{\gamma+x}{1+x \gamma}\right) \\
& =\frac{1}{\left(1+x^{2}\right)^{j}} \sum_{k=0}^{2 j} f_{k}(\gamma+x)^{k}(1+x \gamma)^{2 j-k} \\
& =\sum_{k=0}^{2 j} \tilde{f}_{k}[x] \gamma^{k} .
\end{aligned}
$$

Such a rotation leaves the moments $W^{(q)}$ invariant. We now want to find a value $x_{0}$ so that the combined action $\mathcal{F}_{2} \mathcal{R}_{x_{0}}$ increases these moments by a finite amount when applied to $\psi(\gamma)$.

We consider the coefficient

$$
\tilde{f}_{0}[x]=\frac{1}{\left(1+x^{2}\right)^{j}} \sum_{k=0}^{2 j} f_{k} x^{k}
$$

of the rotated Bargmann function as a function of $x$. Since all the coefficients $f_{k}$ are positive and $\tilde{f}_{0}(x) \rightarrow 0$ when $x \rightarrow \infty, \tilde{f}_{0}[x]$ has a maximum value for some $x_{\max } \geq 0$. As $\psi(x)$ is not a coherent state, some $f_{k}$ with $k>0$ does not vanish and we have $x_{\max }>0$. Also for the rotated Bargmann function, $\mathcal{R}_{x_{\max }} \circ \psi(x)$, there exist some nonvanishing coefficients, $\tilde{f}_{k}\left[x_{\max }\right]$, since rotating a non-coherent state results still in a non-coherent state. At least one of the non-vanishing coefficients must be negative $f_{k}\left[x_{\max }\right]<0$. If no coefficients were negative, there would be a rotation $\mathcal{R}_{x}$ that increases $f_{0}[x]$ further.

Now define

$$
\left|\psi^{\prime}\right\rangle=\mathcal{F}_{2} \circ \mathcal{R}_{x_{\max }}(|\psi\rangle) .
$$

We have then have $W_{\left|\psi^{\prime}\right\rangle}^{(q)} \geq W_{|\psi\rangle}^{(q)}$. Only if the signs of coefficients of $\mathcal{R}_{x_{\max }} \circ \psi(\gamma)$ are related according to $\tilde{f}_{k}\left[x_{\max }\right]=\left|\tilde{f}_{k}\left[x_{\max }\right]\right|(-1)^{k}$ we have $W_{\left|\psi^{\prime}\right\rangle}^{(q)}=W_{|\psi\rangle}^{(q)}$ according to lemma 1. In this case one may replace the rotation $\mathcal{R}_{x_{\max }}$ by $\mathcal{R}_{x_{\max }-\epsilon}$ for sufficiently small $\epsilon$. For small $\epsilon>0$ we have $\tilde{f}_{1}\left[x_{\max }-\epsilon\right]>0$ since $\tilde{f}_{0}[x]$ has an isolated maximum at $x=x_{\max }$ and $\frac{d}{d x} \tilde{f}_{0}[x]=\tilde{f}_{1}[x]$. We can chose $\epsilon$ small enough such that the negative coefficients remain negative (we have shown above that at least one 
coefficient is negative). Since now $\tilde{f}_{0}\left[x_{\max }-\epsilon\right]>0$ and $\tilde{f}_{1}\left[x_{\max }-\epsilon\right]>0$ the relation $\tilde{f}_{k}\left[x_{\max }-\epsilon\right] \neq\left|\tilde{f}_{k}\left[x_{\max }-\epsilon\right]\right|(-1)^{k}$ cannot be fullfilled and we have $W_{\left|\psi^{\prime}\right\rangle}^{(q)}>W_{|\psi\rangle}^{(q)}$.

This proof shows what a possible future proof of Lieb's conjecture on $S_{|\psi\rangle}$ might be. With $S_{|\psi\rangle}=2 j-\sum_{n=2}^{\infty} \frac{Y_{|\psi\rangle}^{(n)}}{n(n+1)}$ Lieb's conjecture is equivalent to the statement that all dual moments $Y^{(n)}$ are maximalized by coherent states for $n=2,3, \ldots$. For example, if it can be shown that $Y_{\mathcal{F}_{2}\left(\left|\psi^{\prime}\right\rangle\right)}^{(n)} \geq Y_{|\psi\rangle}^{(n)}$ for $n=2,3, \ldots$ an analogous proof can be given for Lieb's conjecture.

It might be also interesting to consider the delocalized pure states characterized by the maximal entropy. In the space of mixed quantum states, the maximally delocalized state is proportional to the identity matrix, $\rho_{*}:=\mathbb{I} /(2 j+1)$, for which $S_{\rho_{*}}^{(q)}=\log (2 j+1)$ and $R_{\rho_{*}}=2 j+1$. However, the maximal entropy $S^{\max }$ is in general unknown, if one looks for a maximum in the space of all pure states. Lee conjectured [32] that this maximum is achieved for pure states with possibly regular distribution of all $2 j$ zeros of the Husimi function on the sphere. Such a distribution of zeros is easy to specify for $2 j=4,6,8,12,20$, which correspond to the Platonic polyhedra [17], and it seems to be plausible that these states provide the maxima for all the generalized entropies $S^{(q)}$.

3.5. Generalizations to other compact phase spaces. We have introduced generalized Lieb-Wehrl entropies and discussed their minimal values (Lieb-Wehrl conjecture). The above results, obtained for the Bloch sphere $S^{2}$ and the $S U(2)$ spin coherent states, may be generalized for classical phase spaces associated with other (compact) Lie groups such as $S U(d) ; d \geq 2$. In that case the classical phase spaces are equivalent to complex projective spaces, $\mathbb{C} P^{d-1}$, and the dimension of the Hilbert space equals 0 冏 $N=\operatorname{dim} V=\left(\begin{array}{c}m+d-1 \\ m\end{array}\right)$, with $m=1,2, \ldots$ The Wehrl entropy of an $S U(d)$ coherent state equals [39, 40 ]

$$
S_{\mathrm{coh}}=m[\Psi(m+d)-\Psi(m+1)],
$$

which for $d=2$ reduces to $(N-1) / N$ as desired. Our generalization of the LiebWehrl conjecture stated that this value gives the minimal Wehrl entropy for all pure states corresponding to the classical phase space $\mathbb{C} P^{d-1}$. The methods we used to give proofs of some special cases of this conjecture on the sphere should be applicable in that case as well.

\section{RANDOM PURE STATES}

4.1. Rényi-Wehrl entropies for random states. Spin coherent states are localized in phase space as much as allowed by the uncertainty principle and are characterized by the minimal Rényi-Wehrl entropies. Let us emphasize that for $j \gg 1$ the pure states exhibiting Wehrl entropy of the order of $S_{\min }$ are not typical. In a typical situation the density of the zeros of the Bargmann or Husimi function is close to uniform on the sphere [36], and the Wehrl entropy of such delocalized pure states is found to be large.

In order to analyze, to what extend the numbers obtained in (36) are small, we compute the mean entropies averaged over the ensemble of random pure states. A random pure state $|\Phi\rangle$, can be generated according to the natural uniform measure

\footnotetext{
${ }^{6}$ This is true for the physically important class of irreducible representations of the group $S U(d)$ on Hilbert spaces $V$ [26].
} 
on the space of pure states by taking $|\Phi\rangle=U|\kappa\rangle$, where $|\kappa\rangle=|j\rangle$ is the reference state and $U \in U(N)$ with $N=2 j+1$ is a random unitary matrix distributed according to the Haar measure $d \mu(U)$. Such random matrices pertain to the circular unitary ensemble (CUE) [37], often used to describe quantum chaotic systems [38]. We compute the mean Rényi-Wehrl entropies of $N$-dimensional pure states, $\left\langle S^{(q)} q\right\rangle$ by taking the average with respect to this measure. Let us start computing the mean values of the $q$-th moments

$$
\left\langle W^{(q)}\right\rangle:=\int_{U(N)}\left(N \int_{S^{2}}\left[\mathcal{H}_{|\Phi\rangle}(\gamma)\right]^{q} d \mu(\gamma)\right) d \mu(U)
$$

Since $\mathcal{H}_{|\Phi\rangle}(\gamma)=|\langle\Phi \mid \gamma\rangle|^{2}=\left|\left\langle j\left|U^{-1} \mathcal{R}(\gamma, 0)\right| j\right\rangle\right|^{2}$ one may interchange the order of integration and use the invariance of the Haar measure [39]. Putting $V:=$ $U^{-1} \mathcal{R}(\gamma, 0)$ we conclude that

$$
\left\langle W^{(q)}\right\rangle=N \int_{U(N)}|\langle j|V| j\rangle|^{2 q} d \mu(V)
$$

since $\int d \mu(\gamma)=1$.

This average may be computed based on earlier results of Kuś et al. [1] or Jones 40. We obtain $\left\langle W^{(q)}\right\rangle=N \Gamma(N) \Gamma(q+1) / \Gamma(q+N)$, which substituted into the definition leads to

$$
\left\langle S^{(q)}\right\rangle_{N}=\frac{1}{1-q} \ln \left[\Gamma(N+1) \frac{\Gamma(q+1)}{\Gamma(q+N)}\right] .
$$

Thus the mean Wehrl participation number of a typical random state is

$$
R^{\mathrm{rand}}=e^{S_{2}}=\frac{N+1}{2} .
$$

In the limit $q \rightarrow 1$ formula (59) gives the mean Wehrl entropy of a random pure state [39]

$$
\langle S\rangle_{N}=\Psi(N+1)-\Psi(2)=\sum_{n=2}^{N} \frac{1}{n},
$$

where $\Psi(x)=\Gamma^{\prime}(x) / \Gamma(x)$ denotes the digamma function. Thus the mean Wehrl entropy of a random state is equal to the Shannon entropy [40] of its expansion in a relatively random basis 40, 14. The mean Wehrl entropy was used to obtain bounds for dynamical entropies characterizing the properties of quantum maps 39. 41, 42]. Note that another normalization of the coherent states used in [39], leads to results shifted by a constant $-\ln N$. These normalization allows one for a direct comparison between the entropies describing the states of various $N$.

In the asymptotic limit $N \rightarrow \infty$ the mean entropy $\langle S\rangle_{N}$ behaves as $\ln N+\gamma-1 \approx$ $\log N-0.42278$, which is close to the maximal possible Wehrl entropy for mixed states $S_{\rho_{*}}=\log N$. Here $\gamma$ denotes the Euler constant.

Let us mention that the above technique of computing the averages over the CUE does not work for the unitary symmetric matrices of circular orthogonal ensemble, (COE), since writing $V:=\left(U U^{T}\right)^{-1} \mathcal{R}_{\gamma}$ one cannot perform the average in (58). The mean Wehrl entropy of a random 'symmetric' state, $\left|\Phi_{s}\right\rangle:=U^{T} U|j\rangle$ differs therefore from the Shannon entropy $S_{o}$ of an eigenvector of a symmetric random unitary matrix typical of COE, $S_{o}=\Psi(N / 2+1)-\Psi(3 / 2) \sim \ln N+\gamma+\ln 2-2 \approx \ln N-0.7296$ [40. Numerical computations show that the mean Wehrl entropy of random vectors 
$\left|\Phi_{s}\right\rangle$ is slightly smaller than the entropy of $|\Phi\rangle$ given by (61), and this difference vanishes in the asymptotic limit $N \rightarrow \infty$ 17].

4.2. Distance between Husimi functions and uniform distribution. The theory of quantum ergodicity deals with the semiclassical properties of eigenfunctions of a Laplace operator. The Shnirleman theorem says that the expectation values of quantum observables in eigenstates tend to the mean values of the corresponding classical quantities if the classical system is ergodic 433. In the semiclassical limit almost all eigenfunctions of quantum chaotic billiards tend, in a weak sense, to the measures covering uniformly entire configuration space available (the domain of the billiard) [44. An analogous statement concerning the phase space implies that for almost all eigenstates the corresponding Wigner (or Husimi) distributions condensate uniformly on the energy surface.

We may thus expect that the Husimi distribution of a random eigenstate will tend to the uniform distribution, $(2 j+1) \mathcal{H}_{*}(\gamma)=1$ in the limit $j \rightarrow \infty$. Since strong convergence is excluded by the presence of the $2 j$ zeros of the Husimi distribution for any pure state, we will consider weak convergence only. To characterize this convergence quantitatively we introduce the $L_{2}$ distance

$$
L_{2}\left(\mathcal{H}_{|\Phi\rangle}(\gamma), \mathcal{H}_{*}(\gamma)\right):=\left((2 j+1) \int_{S^{2}}\left[\mathcal{H}_{|\Phi\rangle}(\gamma)-\mathcal{H}_{*}(\gamma)\right]^{2} d \mu(\gamma)\right)^{1 / 2} .
$$

We may express the distance to the uniform distribution by the Wehrl participation number (16)

$$
L_{2}^{2}\left(\mathcal{H}_{|\Phi\rangle}, \mathcal{H}_{*}\right)=\frac{2 j+1}{4 \pi} \int_{0}^{2 \pi} d \varphi \int_{0}^{\pi} \sin \vartheta d \vartheta\left[|\langle\gamma \mid \Phi\rangle|^{4}-\frac{1}{(2 j+1)^{2}}\right]=\frac{1}{R}-\frac{1}{2 j+1} .
$$

Using the previous result $(60)$ we get

$$
\left\langle L_{2}^{2}\left(\mathcal{H}_{|\Phi\rangle}, \mathcal{H}_{*}\right)\right\rangle=\frac{2 j}{(2 j+1)(2 j+2)} \rightarrow 0 \quad \text { as } \quad j \rightarrow \infty,
$$

where the average is taken over the natural, unitarily invariant, measure on the manifold of pure states.

In other words, in the semiclassical limit $(j \rightarrow \infty)$ the Husimi distribution of a typical random state tends to the uniform distribution of the sphere in the weak sense. On the other hand, applying (16) we see that the analogous distance for the localized coherent state tends to a constant

$$
L_{2}^{2}\left(\mathcal{H}_{\mathrm{coh}}, \mathcal{H}_{*}\right)=\frac{2 j}{4 j+2} \rightarrow \frac{1}{2}, \quad \text { as } \quad j \rightarrow \infty,
$$

which emphasizes the intuitive fact that the coherent states are exceedingly nontypical in the space of pure states of a large dimensional Hilbert space.

\section{Concluding Remarks}

The Wehrl entropy of a pure quantum state may be considered as a useful measure of its localization in classical phase space. In contrast with the so-called 'eigenvector statistics', it is defined without any ambiguity, provided the quantum problem admits to introduce the set of coherent states distinguished by the

\footnotetext{
${ }^{7}$ In this limit $p(\gamma)=(2 j+1) \mathcal{H}(\gamma)$ may be interpreted as a classical probability distribution on the sphere.
} 
corresponding classical dynamics. In the analogy to the well known notion of the Rényi entropies [45], we defined and investigated the Rényi-Wehrl entropies, which may serve as complementary measures of localization. Although the family of generalized entropies is parametrized by the continuous real Rényi parameter $q$, the generalized entropies are most easily evaluated for integer values of $q$.

Every pure state of a $N$-dimensional Hilbert space may be uniquely represented by the set of $N-1$ zeros of its Husimi (Bargmann) functions defined by the family of coherent states. For the physically most relevant case of the $S U(2)$ coherent states, any state can be represent by $N=2 j$ indistinguishable points on the sphere (which may coalesce) [29, 30, 31]. In this stellar representation the coherent states are distinguished by being the only states, for which all zeros are located in a single point. It is thus natural to expect, that coherent states mimimize the Wehrl entropy, as conjectured by Lieb [22].

Not being able to prove this conjecture in its full glory, in this work we:

i ) have shown that coherent states provide local minima (maxima) of the generalized entropies (moments) for any value of the Rényi parameter $q>0$;

ii ) have proved the generalized Lieb conjecture for integer values $q=2,3, \ldots$.

To achieve these goals we introduced and used the notion of (non-unitary) entropyreducing maps in the space of pure states, which might be helpful in further attempts to prove the Lieb conjecture. Moreover, in this paper we analyzed the random pure states and computed the values of generalized Wehrl entropies averaged over the natural, unitarily invariant measure in the manifold of pure states of $N$-dimensional Hilbert space.

We are indebted to Wojciech Słomczyński for numerous suggestions, long lasting interaction related to this project and reading of the manuscript. We have also enjoyed discussions with Christoffer Manderfeld and Marek Kuś. This work has been supported by the Minerva Foundation and the Polish grant of Komitet Badań Naukowych number 2P03B 07219.

\section{Appendix A. Rotations of Bargmann functions}

Rotations of a state $|\psi\rangle$ by some $\mathcal{R} \in S U(2)$ corresponds to transformations of the corresponding Bargmann and Husimi function. In the defining representation of $S U(2)$ by $2 \times 2$ matrices $(j=1 / 2)$ almost any rotation $\mathcal{R} \in S U(2)$ (with respect to Haar measure) can be written as in the form

$$
\mathcal{R}_{1 / 2}(\alpha, \phi)=\left(\begin{array}{cc}
\frac{e^{i \frac{\phi}{2}}}{\sqrt{1+|\alpha|^{2}}} & -\frac{\bar{\alpha} e^{-i \frac{\phi}{2}}}{\sqrt{1+|\alpha|^{2}}} \\
\frac{\alpha e^{\frac{\phi}{2}}}{\sqrt{1+|\alpha|^{2}}} & \frac{e^{-i \frac{\phi}{2}}}{\sqrt{1+|\alpha|^{2}}}
\end{array}\right)
$$

for some complex $\alpha$ and angle $\phi$. This looks much nicer using the Gaussian decomposition

$$
\mathcal{R}(\alpha, \phi)=\left(\begin{array}{cc}
1 & 0 \\
\alpha & 1
\end{array}\right)\left(\begin{array}{cc}
\left(1+|\alpha|^{2}\right)^{-1 / 2} e^{i \frac{\phi}{2}} & 0 \\
0 & \left(1+|\alpha|^{2}\right)^{1 / 2} e^{-i \frac{\phi}{2}}
\end{array}\right)\left(\begin{array}{cc}
1 & -\bar{\alpha} e^{-i \phi} \\
0 & 1
\end{array}\right)
$$

In the general case of the $2 j+1$ dimensional Hilbert space $V_{j}$ the rotation $\mathcal{R}(\alpha, \phi)$ is represented by the operator

$$
\mathcal{R}_{j}(\alpha, \phi)=e^{\alpha J_{-}} e^{\left(i \phi-\log \left(1+|\alpha|^{2}\right)\right) J_{z}} e^{-\bar{\alpha} J_{+}} .
$$

We omitted the index $j$ of this operator throughout the text. 
RÉNYI-WEHRL ENTROPIES AS MEASURES OF LOCALIZATION IN PHASE SPACE 17

Rotating a coherent state gives another coherent state (up to a normalization constant and a phase factor).

$$
\begin{aligned}
\mathcal{R}(\alpha, \phi) \mid \gamma)= & \exp \left((\mathcal{R}(\alpha, \phi) \circ \gamma) J_{-}\right) \times \\
& \times \exp \left(\left[i \phi+\log \left(\frac{\left(1-\bar{\alpha} \gamma e^{-i \phi}\right)^{2}}{1+|\alpha|^{2}}\right)\right] J_{z}\right)|j\rangle_{j} \\
= & \left.\frac{\left(e^{i \phi}-\bar{\alpha} \gamma\right)^{2 j}}{\left(1+|\alpha|^{2}\right)^{j}} e^{-i \phi j} \mid \mathcal{R}(\alpha, \phi) \circ \gamma\right),
\end{aligned}
$$

where

$$
\mathcal{R}(\alpha, \phi) \circ \gamma=\frac{\gamma+\alpha e^{i \phi}}{e^{i \phi}-\bar{\alpha} \gamma} .
$$

If a rotation acts on a state $|\psi\rangle \mapsto \mathcal{R}(\alpha, \phi)|\psi\rangle$ its Bargmann function transforms like

$$
\left.\psi(\gamma)=\langle\psi| \gamma) \mapsto \psi^{\prime}(\gamma)=\langle\mathcal{R}(\alpha, \phi) \psi| \gamma\right)=\left\langle\psi\left|\mathcal{R}(\alpha, \phi)^{\dagger}\right| \gamma\right)
$$

where

$$
\mathcal{R}(\alpha, \phi)^{\dagger}=\mathcal{R}\left(-\alpha e^{i \phi},-\phi\right) .
$$

The rotated Bargmann function thus is

$$
\psi^{\prime}(\gamma)=\left\langle\psi\left|\mathcal{R}\left(-\alpha e^{i \phi},-\phi\right)\right| \gamma\right)=\frac{(1+\bar{\alpha} \gamma)^{2 j}}{\left(1+|\alpha|^{2}\right)^{j}} e^{-i \phi j} \psi\left(\mathcal{R}\left(-\alpha e^{i \phi},-\phi\right) \circ \gamma\right)
$$

In the stellar decomposition it is obvious that the rotation of the state corresponds to rotation of the zeroes of its Bargmann function

$$
\psi(\gamma)=\mathcal{N}^{-1} \prod_{k=1}^{2 j}\left(\gamma-\gamma_{k}^{(0)}\right) \mapsto \psi^{\prime}(\gamma)=\mathcal{N}^{\prime-1} \prod_{k=1}^{2 j}\left(\gamma-\mathcal{R}(\alpha, \phi) \circ \gamma_{k}^{(0)}\right)
$$

where

$$
\mathcal{N}^{\prime}=\mathcal{N}\left(1+|\alpha|^{2}\right)^{j} e^{-i \phi j} \prod_{k=1}^{2 j}\left(1-\gamma_{k}^{(0)} \bar{\alpha} e^{-i \phi}\right)^{-1}
$$

These transformations are well behaved if some zeros are on the south-pole $\left|\gamma_{k}^{(0)}\right| \rightarrow \infty$.

\section{REFERENCES}

1. M. Kuś, J. Mostowski and F. Haake, J. Phys. A 21, L1073 (1988).

2. O. Bohigas, in: M.-J. Giannoni, A. Voros and J. Zinn-Justin (Eds.), Chaos and Quantum Physics, Les Houches Summer School, Session LII, North-Holland, Amsterdam, 1991.

3. F. Izrailev, Phys. Rep. 129, 299 (1990).

4. F. Haake and K. Życzkowski, Phys. Rev. A 42, 1013 (1990).

5. G. Casati, I. Guarneri, F. Izrailev and R. Scharf, Phys. Rev. Lett. 64, 5 (1990).

6. E. Heller, Phys. Rev. A 35, 1360 (1987).

7. A. Perelomov, Generalized Coherent States and their Applications, Springer, Berlin, 1986.

8. R. Gilmore, Ann. Phys. 74, 391 (1972).

9. K. Husimi, Proc. Phys. Math. Soc. Jpn. 22, 264 (1940).

10. K. Takahashi, J. Phys. Soc. Jap. 55, 762 (1986).

11. K. Życzkowski, Phys. Rev. A 35, 3546 (1987).

12. A. Wehrl, Rev. Mod. Phys. 50, 221 (1978).

13. A. Wehrl, Rep. Math. Phys. 30, 119 (1991). 
14. M. Kuś and K. Życzkowski, Phys. Rev. A 44, 956 (1991).

15. D. Weinmann, S. Kohler, G.-L. Ingold and P. Hänggi, Ann. Phys. (Leipzig) 8, SI-277 (1999).

16. I. Varga, E. Hofstetter and J. Pipek, Phys. Rev. Lett. 82, 468 (1999).

17. K. Życzkowski, Physica E 9, 583 (2001).

18. A. Sugita and H. Aiba, LANL e-print hlin.CD/0106012.

19. R. Glauber, Phys.Rev. 130, 2529 (1963).

20. R. Glauber, Phys.Rev. 131, 2766 (1963).

21. E. G. G. Sudarshan, Phys. Rev. Lett 10, 277 (1963).

22. E. H. Lieb, Comm. Math. Phys. 62, 35 (1978).

23. J. M. Radcliffe, J. Phys. A 4, 313 (1971).

24. F. T. Arecchi, E. Courtens, R. Gilmore, and H. Thomas, Phys. Rev. A 6, 2211 (1972).

25. D. M. Gitman and A. L. Shelepin, J. Phys. A 26, 313 (1993).

26. S. Gnutzmann and M. Kuś, J. Phys. A 31, 9871 (1998).

27. K. Nemoto, J. Phys. A 34, 2051 (2000).

28. R. Delbourgo, J. Phys. A 10, 1837 (1977).

29. E. Majorana, Nuovo Cimento 9, 43 (1932).

30. H. Bacry, J. Math. Phys. 15, 1686 (1974).

31. R. Penrose, The Emperor's New Mind, Oxford University Press, Oxford, 1989

32. C.-T. Lee, J. Phys. A 21, 3749 (1988).

33. P. Schupp, Comm. Math. Phys. 207, 481 (1999).

34. H. Scutaru, preprint FT-180 (1979) and LANL e-print math-ph/9909024.

35. K. Życzkowski and W. Słomczyński, J. Phys. A (2001) in press and LANL e-print quant-ph/0008016.

36. P. Lebœuf and A. Voros, J. Phys. A 23, 1765 (1990).

37. M. L. Mehta, Random Matrices, 2nd ed., Academic, New York, 1991.

38. F. Haake Quantum Signatures of Chaos, 2nd ed., Springer, 2000.

39. W. Słomczyński and K. Życzkowski, Phys. Rev. Lett. 80, 1880 (1998).

40. K. R. W. Jones, J. Phys. A 23, L1247 (1990).

41. B. Mirbach and H. J. Korsch, Phys. Rev. Lett. 75, 362 (1995).

42. B. Mirbach and H. J. Korsch, Ann. Phys. (N.Y.) 265, 80 (1998).

43. A. I. Shnirelman, Usp. Math. Nauk. 29, 181 (1974).

44. S. Zelditch and M. Zworski, Comm. Math. Phys. 175, 673 (1996).

45. A. Rényi, in. Proc. Fourth. Berkeley Symp. Math. Stat. Prob. 1960, Vol. I, p.547, University of California Press, Berkeley, 1961.

Sven Gnutzmann, Department of Physics of Complex Systems, The Weizmann InstiTUte of Science, Rehovot, IsRael

E-mail address: sven@gnutzmann.net

Karol Życzkowski, Centrum Fizyki Teoretycznej, Polska Akademia Nauk, Warszawa, Polska, on leave from Instytut Fizyki, Uniwersytet Jagielloński, Kraków, Poland

E-mail address: karol@theta1.cft.edu.pl 